\title{
Avaliação da eficácia de uma oficina para qualificação docente em Aprendizagem
}

\section{Baseada em Equipes}

\author{
Evaluating a workshop model effectiveness for faculty training in Team-Based Learning \\ Evaluación de la eficacia de un taller para la calificación docente en el Aprendizaje Basado en
}

Equipo

Recebido: 18/07/2021 | Revisado: 25/07/2021 | Aceito: 28/07/2021 | Publicado: 04/08/2021

Mário Roberto Tavares Cardoso de Albuquerque
ORCID: https://orcid.org/0000-0003-2962-2754
Universidade do Estado do Pará, Brasil.
E-mail: drmarioalbuquerque@ gmail.com
Nara Macedo Botelho
ORCID: https://orcid.org/0000-0003-1781-0133
Universidade do Estado do Pará, Brasil.
E-mail: narambotelho@ gmail.com
Milena Coelho Fernandes Caldato
ORCID: https://orcid.org/0000-0002-7077-8470
Universidade do Estado do Pará, Brasil.
E-mail: milenacaldato@ hotmail.com
Ana Caroline Guedes Souza Martins
ORCID: https://orcid.org/0000-0001-7185-8520
Universidade do Estado do Pará, Brasil.
E-mail: carolguedes.devs@ hotmail.com
Cybelle Cristina Pereira Rodrigues
ORCID: https://orcid.org/0000-0003-3351-5257
Centro Universitário do Estado do Pará, Brasil.
E-mail: cybellecpereira@ gmail.com

\section{Resumo}

Este estudo teve como objetivo avaliar a eficácia de uma oficina de qualificação docente em Aprendizagem Baseada em Equipes (ABE) com docentes do curso de medicina de uma instituição privada de ensino superior de Belém/PA/Brasil. Tratou-se de uma pesquisa-ação por meio de questionários para avaliar o conhecimento prévio dos docentes acerca da metodologia da $\mathrm{ABE}$ antes e após as oficinas propostas. Os resultados demonstraram que a taxa de acertos quanto ao número de etapas da metodologia subiu de $15,62 \%$ para 96,9\%. Em relação a quais são as etapas da ABE, os acertos subiram de 3,12\% para 87,5\%. Quanto ao tempo destinado para cada etapa, as respostas corretas subiram de 3,12\% para $68,7 \%$. Ressalta-se que $96,9 \%$ dos entrevistados referiram compreender os benefícios da $\mathrm{ABE}$ após as oficinas realizadas e, destes, 53,12\% não compreendiam anteriormente. Isso permite afirmar que as oficinas além de qualificarem os docentes, também foram capazes de sensibilizá-los quanto aos benefícios dessa estratégia educacional no processo de ensino-aprendizagem. Tais dados apontam para o sucesso das oficinas, permitindo assim que os participantes possam aplicar essa estratégia de ensino com maior segurança e assertividade em sua rotina docente. Constatou-se, portanto, que a tecnologia educacional desenvolvida é uma ferramenta válida para implementação no desenvolvimento docente de outras instituições de ensino. Espera-se que esse trabalho possa contribuir para a aprendizagem acerca da ABE e possa torná-la, de forma a estimular docentes e instituições a introduzirem essa valiosa ferramenta em suas realidades.

Palavras-chave: Autoaprendizagem como assunto; Educação médica; Docentes de medicina; Ensino.

\begin{abstract}
This study aimed to evaluate the effectiveness of a teacher qualification workshop in Team-Based Learning (TBL) with medical professors from a private higher education institution in Belém/PA/Brazil. It was an action research through questionnaires to assess the prior knowledge of teachers about the TBL methodology before and after the proposed workshops. The results showed that the rate of correct answers regarding the number of steps in the methodology rose from $15.62 \%$ to $96.9 \%$. Regarding the stages of the TBL, the correct answers rose from $3.12 \%$ to $87.5 \%$. As for the time allocated for each stage, the correct answers rose from $3.12 \%$ to $68.7 \%$. It is noteworthy that $96.9 \%$ of respondents reported understanding the benefits of TBL after the workshops held and, of these, $53.12 \%$ did not understand it before. This allows us to state that the workshops, in addition to qualifying teachers, were also able to sensitize them about the benefits of this educational strategy in the teaching-learning process. Such data point to the success of the workshops, thus allowing the participants to apply this teaching strategy with greater security and
\end{abstract}


assertiveness in their teaching routine. It was found, therefore, that the educational technology developed is a valid tool for implementation in the development of teachers in other educational institutions. It is hoped that this work can contribute to learning about TBL and can make it, in order to encourage teachers and institutions to introduce this valuable tool in their realities.

Keywords: Active learning; Medical education; Medical faculty; Teaching.

\section{Resumen}

Este estudio tuvo como objetivo evaluar la efectividad de un taller de capacitación docente en Aprendizaje Basado en Equipos (ABE) con profesores de medicina de una institución privada de educación superior en Belém/PA/Brasil. Se trató de una investigación-acción través de cuestionarios para evaluar el conocimiento previo de los docentes sobre la metodología ABE antes y después de los talleres propuestos. Los resultados mostraron que la tasa de aciertos con respecto al número de pasos en la metodología aumentó de 15,62\% a 96,9\%. En cuanto a las etapas del ABE, las respuestas correctas pasaron del 3,12\% al 87,5\%. En cuanto al tiempo asignado a cada paso, las respuestas correctas pasaron del 3,12\% al 68,7\%. Es de destacar que el 96,9\% de los encuestados informó comprender los beneficios de la ABE después de los talleres realizados y, de estos, el 53,12\% no lo entendió antes. Esto permite afirmar que los talleres, además de capacitar a los docentes, también lograron sensibilizarlos sobre los beneficios de esta estrategia educativa en el proceso de enseñanza-aprendizaje. Dichos datos apuntan al éxito de los talleres, permitiendo así a los participantes aplicar esta estrategia de enseñanza con mayor seguridad y asertividad en su rutina docente. Se encontró que la tecnología educativa desarrollada es una herramienta válida para su implementación en el desarrollo docente de otras instituciones educativas. Se espera que este trabajo pueda contribuir al aprendizaje del ABE y pueda hacerlo, con el fin de animar los docentes e instituciones a introducir esta valiosa herramienta en sus realidades.

Palabras clave: Autoaprendizaje como asignatura; Educación médica; Profesores de medicina; Enseñanza.

\section{Introdução}

A Aprendizagem Baseada em Equipes (ABE) é uma estratégia de ensino que se caracteriza como um método dinâmico de aprender, pois é precedida de uma etapa individual e, em seguida em grupo, para reflexão, sendo este momento em que ocorre a motivação e integração dos alunos, através do compartilhamento de informações, materializando uma importante ferramenta contra o desinteresse do aluno pelo aprendizado (Bandeira, Silva \& Vilela, 2017).

É comum aos estudantes relatarem que a utilização da ABE possibilita que as aulas sejam mais dinâmicas, participativas, produtivas, e o público alvo sente maior estímulo para realização do estudo e troca de conhecimento com os membros do grupo. Ademais, a ABE consiste em uma ferramenta pedagógica que pode ser adaptada a vários contextos e conteúdos, permitindo o pensamento crítico, resolução de problemas, interação entre discentes e docentes, além de estimular o trabalho em equipe (Bandeira, Silva \& Vilela, 2017).

Dessa forma, a aceitação de alguns alunos pelas metodologias ativas é um processo gradual. No entanto, a maioria já considera as metodologias ativas como uma importante ferramenta para a formação de um profissional mais crítico, reflexivo e confiante. Assim, a ABE tem se caracterizado como uma ferramenta pedagógica de sucesso por promover o crescimento acadêmico e proporcionar o autoaprendizado, potencializando habilidades, como o trabalho em equipe (Albuquerque et al., 2021).

Além disso, a ABE tem sido utilizada como um método eficiente de avaliação, em que é de fundamental importância fazer uma análise crítica constante para que as práticas do ensino tradicional não sejam supervalorizadas em detrimento das práticas ativas de aprendizagem. Assim, os docentes devem receber formação permanente a fim de se sentirem confortáveis para mudar sua práxis (Cunha, Ramsdorf \& Bragato, 2019).

Em experiência conduzida na Malásia, a aplicação da ABE tornou os alunos mais ativos, motivados e com melhora da performance em testes escritos, sugerindo que esta estratégia incrementa a qualidade do ensino e otimiza os resultados de aprendizagem (Ismail, 2016). Mesmas conclusões foram observadas no ensino de microbiologia na Índia (Harakuni et al., 2015) e em escola médica da Arábia Saudita (Ibrahim, 2020).

Já no tocante aos docentes, a maioria não conhece este método, e assim julgam conhecer, quando são indagados não conhecem as etapas que compõe este método. Cerca de $50 \%$ dos docentes do ensino superior da saúde relataram nunca terem 
participado de atividades em ABE, 75\% nunca realizaram qualificações prévias sobre a metodologia e 96,87\% sentem necessidade de capacitações acerca da metodologia. A maioria dos profissionais não se consideram qualificados para realizar tais atividades em $\mathrm{ABE}(62,5 \%)$ e nem reconheciam o seu benefício no processo de ensino-aprendizagem (Albuquerque, Martins, Caldato \& Botelho, 2021).

Contudo, ainda se percebe que a mesma é subutilizada nos cursos de graduação e, especialmente, pós-graduação em Medicina (Balwan et al., 2015) quando comparada a outras metodologias ativas como o Problem Based Learning (PBL) e a Problematização, que nos últimos anos têm sido as ferramentas mais adotadas pelos cursos de graduação em Medicina do Brasil com o objetivo de adequação as novas diretrizes curriculares de formação médica (Brasil, 2014).

Além disso, do ponto de vista das instituições de ensino, várias estratégias têm sido consideradas para contemplar o desenvolvimento das competências profissionais. Porém, uma das limitações em algumas delas é a necessidade de turmas ou grupos pequenos e, consequentemente, de mais professores/tutores (Keshmiri et al, 2016) o que eleva os custos para implementação e manutenção dos cursos (Altintas, Altintas \& Caglar, 2014). Quando a turma é grande, tornar o processo de aprendizagem mais dinâmico e significativo torna-se um desafio maior. Nesse contexto a ABE pode ser aplicada com uma proporção que chega a 200 alunos para 1 professor (Echeto et al., 2015), mas também em turmas menores de até 25 alunos (Bollela et al., 2014).

Estudos relatam que $72 \%$ dos artigos incluíam a avaliação de resultados de aprendizagem e que a maioria dos estudos envolviam estudantes de graduação. Cerca de $61 \%$ concluíram que a ABE se constitui de uma técnica instrucional eficaz. No entanto, são escassos os trabalhos que abordem o público docente nessas atividades (Reimschisel et al., 2017).

Até a presente data não foi encontrado nenhum outro estudo semelhante a presente pesquisa, que avalie conhecimento de docentes de Medicina e com formulação de oficinas para a qualificação profissional, o que foi o fator motivador para a realização deste estudo e para que se fosse pensado em um modelo de oficinas para qualificação docente nessa estratégia educacional.

Desta forma, este estudo teve como objetivo avaliar a eficácia de um modelo proposto para qualificação docente em Aprendizagem Baseada em Equipes com docentes do curso de medicina de uma instituição privada de ensino superior de Belém/PA/Brasil.

\section{Metodologia}

Tratou-se de uma pesquisa-ação intervencionista, de abordagem quantitativa. A pesquisa-ação constitui-se em intervenção em uma determinada realidade social e tem características de abordagem longitudinal e prospectiva. (Thiollent, 2013). Na pesquisa quantitativa, as etapas se constituem de observação dos fenômenos; análise dos elementos constitutivos desses fenômenos, e possui finalidade de estabelecer relações quantitativas entre eles; indução de certo número de hipóteses; verificação das hipóteses aventadas por intermédio de experiências; generalização do resultado das experiências para casos similares; confirmação das hipóteses, obtendo-se, a partir delas, leis gerais (Pereira et al., 2018).

Participaram do estudo 32 docentes do curso de medicina de uma Instituição de Ensino Superior (IES) privada em Belém, Pará. O convite foi feito por correio eletrônico através de inscrições utilizando-se o Google Forms®.

Os docentes participaram de duas oficinas de capacitação segundo a metodologia ABE, que ocorreram com intervalo de uma semana entre as etapas. A etapa inicial consistiu em uma explanação acerca dos objetivos e componentes da atividade, com auxílio de recursos tecnológicos audiovisuais.

$\mathrm{Na}$ primeira oficina apresentou-se o estudo aos docentes, com entrega do Termo de Consentimento Livre e Esclarecido (TCLE) e aplicação de questionário contendo 08 (oito) perguntas fechadas sobre a ABE e o perfil profissional objetivando avaliar o conhecimento e experiência prévios dos docentes. Estimou-se uma duração máxima de 15 minutos para 
explicação do projeto e assinatura dos TCLE e mais 15 minutos para preenchimento do questionário. Os questionários foram identificados através de códigos, a fim de manter o sigilo das informações. As variáveis avaliadas incluíam: idade, sexo, titulação e anos de atuação na disciplina dos participantes pesquisados, que serviram posteriormente para equiparar os membros das equipes.

Após a aplicação do questionário inicial, para avaliação do conhecimento prévio dos docentes, foi realizada uma breve explanação acerca das etapas e propostas da $\mathrm{ABE}$, com duração de 30 minutos. Foi realizada uma roda de conversa almejando detectar as principais dificuldades, opiniões e sugestões para implementar a ABE nas atividades práticas diárias, com duração de 60 minutos. Ao final foram entregues dois artigos para estudo dirigido, que serviram de base para as atividades da oficina seguinte que ocorreu uma semana depois no mesmo local e horário.

$\mathrm{Na}$ segunda oficina foi desenvolvida a qualificação sobre o método ABE mediante a leitura prévia dos artigos entregues pelo autor, utilizando as próprias etapas da ABE. Conforme a metodologia preconizada, foi entregue um teste individual com oito questões de múltipla escolha contendo quatro alternativas, com 20 minutos para resolução.

Logo após o grupo foi distribuído de forma intencional, randomizando e equiparando conforme anos de experiência na docência e experiência em ABE, em quatro grupos de oito participantes. É válido ressaltar que a distribuição divergiu do preconizado de número ímpar de integrantes com 5 a 7 participantes cada grupo. Porém isso não afetou a realização da oficina.

$\mathrm{Na}$ etapa seguinte ocorreu a resolução do teste em grupo, com duração de 20 minutos. Na sequência foram exibidas as questões para todos os grupos em slides e foram entregues quatro placas com cinco alternativas para que cada grupo defendesse a sua resposta gerando discussão e feedback imediato pelo autor da pesquisa, com 30 minutos de duração.

$\mathrm{Na}$ terceira etapa correspondente a aplicação dos conceitos, foi resolvido um caso complexo envolvendo a implementação de atividades de ABE na graduação em Medicina. Cada grupo apresentou um planejamento estratégico apontando pontos críticos e possíveis soluções para a problemática proposta, dessa forma aplicando os conceitos teóricos estudados em uma vivência prática como defende a metodologia da ABE. Foram destinados 60 minutos para a realização dessa etapa e cada grupo apresentou seu planejamento em cartolinas para os demais.

Ao final, os docentes resolveram novamente o questionário pré-oficinas objetivando avaliar a retenção de conhecimento após a atividade, com duração prevista de 20 minutos, totalizando 2 horas e 30 minutos para a realização da Oficina 2.

Após a coleta de dados foi elaborado uma planilha eletrônica, para armazenamento dos dados, no software Microsoft Excel® 365. Para representação descritiva dos dados foram elaboradas tabelas, utilizando o Software Microsoft Word 365®. As frequências absolutas e relativas percentual foram descritas para as variáveis categóricas. A análise dos dados foi processada utilizando-se o programa SPSS versão 23. Para comparar as concordâncias e discordâncias entre os pares de observações nos dois momentos (antes e após a oficina de ABE), no qual utilizou-se o teste de McNemar considerando um nível de significância de 5\% (p-valor $<0,05$ ).

A pesquisa foi submetida ao Comitê de Ética em Pesquisa (CEP) do Centro Universitário do Estado do Pará (CESUPA) e aprovada sob o parecer de número 3.054.944. Os participantes selecionados tiveram suas identidades totalmente resguardadas, em respeito à resolução de $\mathrm{N}^{\circ} 466 / 12$ do Conselho Nacional de Saúde (CNS), seguindo os preceitos da Declaração de Helsinki e do Código de Nuremberg.

\section{Resultados}

Quando questionados sobre experiências anteriores (Tabela 1), 50\% dos entrevistados relataram nunca ter se inscrito em atividades de TBL. 75\% nunca haviam se inscrito em habilitações anteriores na metodologia. E 96,87\% sentiram necessidade de se qualificar mais na metodologia. A maioria deles não se considerou habilitado para realizar atividades de 
TBL (62,5\%), nem reconheceu os benefícios da TBL para o processo de ensino-aprendizagem.

Tabela 1: Distribuição dos Participantes das oficinas conforme experiência em ABE.

\begin{tabular}{|c|c|c|c|}
\hline Quesito Avaliado & Sim & Não & Total \\
\hline Já participou de Atividade de ABE & $16(50 \%)$ & $16(50 \%)$ & 32 \\
\hline $\begin{array}{l}\text { Sente necessidade de qualificação no } \\
\text { assunto }\end{array}$ & $31(96,875 \%)$ & $1(3,125 \%)$ & 32 \\
\hline $\begin{array}{l}\text { Já realizou qualificação prévia sobre } \\
\text { ABE }\end{array}$ & $8(25 \%)$ & $24(75 \%)$ & 32 \\
\hline $\begin{array}{l}\text { Se sente capaz de conduzir uma } \\
\text { atividade em ABE? }\end{array}$ & $20(62,5 \%)$ & $12(37,5 \%)$ & 32 \\
\hline $\begin{array}{l}\text { Acredita que a ABE traga benefícios } \\
\text { ao ensino-aprendizagem? }\end{array}$ & $15(46,875 \%)$ & $17(53,125 \%)$ & 32 \\
\hline
\end{tabular}

Fonte: Dados da pesquisa (2020).

Ao avaliar o desempenho individual dos participantes (Tabela 2), estabeleceu-se um ponto de corte de $70 \%$, correspondente a um mínimo de 22 pontos dos 32 pontos totais. Dessa forma, observou-se que 93,75\% dos participantes obtiveram desempenho insatisfatório $(<70 \%)$.

Tabela 2: Desempenho dos participantes no teste individual de garantia do preparo na Oficina 2.

\begin{tabular}{llllll}
\hline Desempenho & $\mathbf{0}$ a 22 pontos & $\begin{array}{l}\mathbf{2 3} \text { a 27 } \\
\text { pontos }\end{array}$ & $\begin{array}{l}\mathbf{2 8} \text { a } 31 \\
\text { pontos }\end{array}$ & 32 pontos & Total \\
\hline Satisfatório $(>70 \%$ do teste $)$ & 0 & $2(6,25 \%)$ & 0 & 0 & $2(6,25 \%)$ \\
\hline Insatisfatório $(<70 \%$ do teste $)$ & $30(93,75 \%)$ & 0 & 0 & 0 & $30(93,75 \%)$ \\
\hline Total & $\mathbf{3 0}(\mathbf{9 3 , 7 5 \% )}$ & $\mathbf{2}(\mathbf{6 , 2 5 \%})$ & $\mathbf{0}$ & $\mathbf{0}$ & $\mathbf{3 2}(\mathbf{1 0 0 \%})$ \\
\hline
\end{tabular}

Fonte: Dados da pesquisa (2020).

Por outro lado, ao avaliar o desempenho dos participantes em grupo (Tabela 3), observou-se que todas as quatro equipes atingiram aproveitamento satisfatório (>70\% de acertos) com uma equipe atingindo acima de $90 \%$ de aproveitamento.

Tabela 3: Desempenho dos participantes no teste em grupo de garantia do preparo na Oficina 2.

\begin{tabular}{lccccc}
\hline Grupo & $\mathbf{0}$ a 22 pontos & $\mathbf{2 3}$ a 27 pontos & 28 a 31 pontos & 32 pontos & Total \\
\hline Grupo 1 & 0 & $1(25 \%)$ & 0 & 0 & $1(25 \%)$ \\
\hline Grupo 2 & 0 & $1(25 \%)$ & 0 & 0 & $1(25 \%)$ \\
\hline Grupo 3 & 0 & 0 & $1(25 \%)$ & 0 & $1(25 \%)$ \\
\hline Grupo 4 & 0 & $1(25 \%)$ & 0 & 0 & $1(25 \%)$ \\
\hline Total & $\mathbf{0}$ & $\mathbf{3 ( 7 5 \% )}$ & $\mathbf{1 ( 2 5 \% )}$ & $\mathbf{0}$ & $\mathbf{4 ( 1 0 0 \% )}$ \\
\hline
\end{tabular}

Fonte: Dados da pesquisa (2020).

Ao comparar as avaliações antes e depois das oficinas, observou-se que a taxa de sucesso nas respostas sobre as fases da metodologia passou de $15,6 \%$ para $96,9 \%$ (Tabela 4). 
Tabela 4: Avaliação do conhecimento dos docentes acertas de quantas etapas são preconizadas na ABE antes e após as oficinas.

\begin{tabular}{ccccc}
\hline $\begin{array}{l}\text { Quantas } \\
\text { Etapas? }\end{array}$ & \multicolumn{2}{c}{ Depois } & \\
\hline Antes & \multicolumn{1}{c}{ Erros } & Acertos & Total & p-valor \\
\hline Erros & $1(3,7 \%)$ & $26(96,3 \%)$ & $27(100 \%)$ & \\
\hline $\begin{array}{c}\text { Acertos } \\
\text { Total }\end{array}$ & $0(0 \%)$ & $5(100 \%)$ & $5(100 \%)$ & $0,0000 *$ \\
\hline $\begin{array}{l}\text { Fonte: Dados da pesquisa, 2020. } \\
(\mathbf{p}<\mathbf{0 , 0 5})\end{array}$ & & $32(96,9 \%)$ & $32(100 \%)$ & \\
\hline
\end{tabular}

Fonte: Dados da pesquisa (2020).

Ao responder sobre quais são as fases do TBL (Tabela 5), a taxa de sucesso passou de 3,1\% para 87,5\%.

Tabela 5: Avaliação do conhecimento dos docentes acertas de quais etapas são preconizadas na ABE antes e após as oficinas.

\begin{tabular}{ccccc}
\hline $\begin{array}{c}\text { Quais } \\
\text { Etapas? }\end{array}$ & \multicolumn{2}{c}{ Depois } & \\
\hline Antes & Erros & Acertos & Total & p-valor \\
\hline Erros & $4(12,9 \%)$ & $27(87,1 \%)$ & $31(100 \%)$ & \\
\hline Acertos & $0(0 \%)$ & $1(100 \%)$ & $1(100 \%)$ & $0,0000^{*}$ \\
\hline Total & $4(12,5 \%)$ & $28(87,5 \%)$ & $32(100 \%)$ & \\
\hline $\begin{array}{l}\text { Fonte: Dados da Pesquisa, 2020. } \\
(\mathbf{p}<\mathbf{0 , 0 5})\end{array}$ & & & $*$ Teste de McNemar \\
\hline
\end{tabular}

Fonte: Dados da pesquisa (2020).

Em relação a quais são as etapas da $\mathrm{ABE}$ (Tabela 6), os acertos subiram de 3,12\% para 87,5\%. No que tange o tempo destinado para cada etapa, as respostas corretas subiram de $3,12 \%$ para $68,7 \%$. Todos esses resultados foram estatisticamente significantes segundo teste de McNemar com $\mathrm{p}$ valor $<0,05$.

Tabela 6: Avaliação do conhecimento dos docentes acertas de qual o tempo correto destinado para cada etapa na ABE antes e após as oficinas.

\begin{tabular}{lcccc}
\hline $\begin{array}{l}\text { Qual o Tempo } \\
\text { Destinado por Etapa? }\end{array}$ & \multicolumn{2}{c}{ Depois } & \\
\hline Antes & Erros & Acertos & Total & p-valor \\
\hline Erros & $10(32,3 \%)$ & $21(67,7 \%)$ & $31(100 \%)$ & $0,0000^{*}$ \\
\hline Acertos & $0(0 \%)$ & $1(100 \%)$ & $1(100 \%)$ & $32(100 \%)$ \\
\hline Total & $10(31,3 \%)$ & $22(68,7 \%)$ & & $*$ Teste de McNemar \\
\hline $\begin{array}{l}\text { Fonte: Dados da pesquisa, 2020. } \\
(\mathbf{p}<\mathbf{0 , 0 5})\end{array}$ & & & \\
\hline
\end{tabular}

Fonte: Dados da pesquisa (2020). 
Ressalta-se que 96,9\% dos entrevistados afirmaram compreender os benefícios da ABE após a realização das oficinas (Tabela 7) e, destes, 53,12\% não compreendiam anteriormente. Este aanho que foi estatisticamente significante com p valor < 0,05 e que permite afirmar que as oficinas além de qualificarem os docentes, também os sensibilizam quanto aos benefícios no processo de ensino-aprendizagem.

Tabela 7: Avaliação da sensibilização dos docentes acerca dos benefícios da ABE no ensino-aprendizagem antes e após as oficinas.

\begin{tabular}{ccccc}
\hline $\begin{array}{l}\text { Entende os Benefícios da } \\
\text { ABE no Ensino- } \\
\text { Aprendizagem? }\end{array}$ & \multicolumn{3}{l}{ Depois } \\
\hline Antes & Não & Sim & Total & p-valor \\
\hline Não & $1(5,9 \%)$ & $16(94,1 \%)$ & $17(100 \%)$ & \\
\hline Sim & $0(0 \%)$ & $15(100 \%)$ & $15(100 \%)$ & $0,0000^{*}$ \\
\hline Total & $1(3,1 \%)$ & $32(96,9 \%)$ & $32(100 \%)$ & \\
\hline $\begin{array}{l}\text { Fonte: Dados de Pesquisa, 2020. } \\
\text { *Teste de McNemar }(\mathbf{p}<\mathbf{0 , 0 5})\end{array}$ & & & \\
\hline
\end{tabular}

Fonte: Dados da pesquisa (2020).

\section{Discussão}

Na presente pesquisa os critérios estabelecidos para a distribuição nos grupos foram: sexo, idade, anos de atuação na docência, titulação e experiência prévia com ABE. Definir o perfil dos docentes se fez necessário pois conforme a metodologia original estabelecida por Michaelsen (2002) no momento de distribuir os participantes em grupos é recomendável que seja feito por critérios e não de forma aleatória, a fim de garantir uma melhor homogeneidade entre as equipes.

Ao serem indagados sobre experiências prévias, $50 \%$ dos entrevistados relataram nunca terem sido participantes de atividades de ABE, 75\% nunca realizaram qualificações prévias sobre a metodologia e 96,87\% sentiam necessidade de capacitações sobre a metodologia. Esses resultados mostram-se relevantes sobretudo para as comissões de desenvolvimento docente da IES sede da pesquisa e das demais, visto que muitas das vezes alguns temas se repetem ou são desinteressantes para o público-alvo, ao passo que a $\mathrm{ABE}$ poderia ser inclusa com grande aceitação e motivação dos profissionais.

Embora os docentes participantes desta pesquisa não tinham tido contato prévio com esta metodologia, Barbiero et al. (2017) aponta para as mudanças que vem ocorrendo no ensino nos cursos de medicina, em que as metodologias de ensino ativas se encontram cada vez mais em destaque no ensino superior, pois possibilitam que o discente deixe de ser apenas ouvinte e passe a ter participação no aprendizado, promovendo uma visão crítica e reflexiva frente ao conteúdo estudado. Tais atributos são fundamentais para um estudante de Medicina, tendo em vista que a atividade profissional que será desenvolvida por ele após a graduação envolve um ambiente de incertezas, tornando-se, então, essencial estimular a formação de um profissional capacitado, sobretudo em buscar por si mesmo novos conhecimentos (Barbiero et al., 2017).

Jost et al. (2017) pontuam que atividades de ABE funcionam mais efetivamente quando conduzidas por profissionais com ampla experiência clínica no assunto a ser trabalhado e capacitados na estratégia educacional. Assim, estes professores conseguem conduzir até 200 alunos sem prejuízos ao processo de ensino-aprendizagem.

Ao avaliar o desempenho individual dos participantes, estabeleceu-se um ponto de corte de 70\%, correspondente a um mínimo de 22 pontos dos 32 pontos totais. Dessa forma, observou-se que $93,75 \%$ dos participantes obtiveram desempenho insatisfatório $(<70 \%)$. Tal dado poderia estar relacionado a má formulação das questões, qualidade dos artigos entregues, tempo de estudo insuficiente, entre outros. Estes itens supramencionados foram abordados no questionário de avaliação das oficinas, porém não foram apontados pelos participantes como fatores dificultadores. 
Contudo, um ponto que pode justificar esse achado é o fato de os participantes não terem realizado o preparo prévio. Esse ponto específico não foi abordado nos questionários propostos e, dessa forma, em experiências futuras é algo a ser considerado. Allen et al. (2013) avaliaram os recursos mais utilizados para preparo prévio no curso de Farmácia nos Estados Unidos, sendo estes: diapositivos em PowerPoint ${ }^{\circledR}$, livros textos e artigos. Os menos fornecidos eram recursos de áudio ou vídeo, que curiosamente eram os preferidos pelos alunos.

Sabe-se que o preparo prévio depende em grande parte do interesse e organização dos alunos em destinar um tempo livre para o estudo com qualidade dos temas. Vários estudos na literatura reportam conclusões semelhantes, em que os alunos não realizam o preparo prévio adequadamente e assim interferindo diretamente no desfecho das atividades (Echeto et al., 2015; Boysen-Osborn et al., 2016).

Como as oficinas foram realizadas com docentes, portanto com vidas profissionais estabelecidas e compromissos diversos, é provável que o tempo de uma semana destinado entre as oficinas não tenha sido adequado para o estudo ou não tenha gerado motivação dos participantes. Curiosamente, foi a mesma dificuldade apontada pelos docentes em suas experiências prévias com alunos da graduação, e também é um fator dificultador importante reportado na literatura.

Bleske et al. (2014) relatam que a complexidade das leituras e o tempo inadequado para que elas sejam integralizadas, o que influencia na satisfação e desempenho dos alunos. Sendo assim, essa é uma etapa, dentro da ABE, que pode ser mais bem estudada e aperfeiçoada com estratégias que garantam a qualidade do preparo prévio.

Por outro lado, ao avaliar o desempenho dos participantes em grupo, observou-se que todas as quatro equipes atingiram aproveitamento satisfatório (>70\% de acertos) com uma equipe atingindo acima de $90 \%$ de aproveitamento. Corroborando os dados globais de revisões sistemáticas sobre ABE em que os desempenhos em grupo superam os melhores desempenhos individuais (Oliveira et al. 2018) e que tornam essa estratégia tão atraente sob a óptica do trabalho em equipe.

Todavia, esse achado nem sempre é universal. Huang et al. (2016) concordam que o conhecimento adquirido com a construção em equipe é mais estável e durável comparado ao adquirido com a escuta dos professores. No entanto, verificaram que a pontuação geral da equipe nem sempre superava a pontuação do melhor membro da equipe.

Os autores creditaram esse fenômeno ao fato de alunos de diferentes grupos acadêmicos compartilharem e discutirem suas ideias uns com os outros, e por vezes eles poderiam chegar a uma resposta incorreta, apoiados pela maioria. No entanto, isso também pode ter ocorrido devido ao fato de apenas uma semana de atividade ser um tempo muito curto para desenvolver a responsabilidade e engajamento das equipes.

Além disso, deve-se pontuar que outros trabalhos sugerem que essa estratégia educacional pode beneficiar somente aqueles alunos que possuem maiores dificuldades de aprendizagem e que precisam ser ajudados pelos outros membros da equipe (Koh et al., 2020).

$\mathrm{Na}$ terceira e última etapa foi entregue um caso complexo, com situação realística envolvendo a implementação da ABE no ensino superior, com 60 minutos para realização, seguido da leitura por todos os grupos das sugestões propostas, o que serviu para que cada um pudesse implementá-las em suas práticas diárias onde a metodologia ainda não é empregada. Por fim, foi entregue novamente o questionário de avaliação do conhecimento sobre ABE, a fim de avaliar se houve incremento de conhecimento após as oficinas.

Ao avaliar os resultados do teste pré e pós-oficinas percebeu-se que a taxa de acertos quanto a todos os questionamentos propostos subiu em valores estatisticamente significantes $(\mathrm{p}<0.05)$, o que permite confirmar que as oficinas foram eficientes para incrementar e qualificar os docentes acerca da metodologia da $A B E$, permitindo assim que os participantes possam aplicar essa estratégia de ensino com maior segurança e assertividade em suas rotinas diárias. Constatouse, portanto, que a tecnologia educacional desenvolvida é uma ferramenta válida para implementação no desenvolvimento docente de outras instituições de ensino que desejem implementá-la em suas matrizes curriculares. 
É importante destacar que nesse trabalho foi avaliado somente a retenção imediata de conteúdo. Sakamoto et al. (2020) constataram que a apreensão de conhecimento de alunos do grupo de ABE foi significativamente maior em comparação com o grupo controle de aulas tradicionais. Contudo, após 30 dias, não houve diferença significativa entre os grupos.

Para Pereira e Afonso (2020) a percepção dos alunos do curso de Fisioterapia, que utilizaram ABE na disciplina de Morfofisiologia foi positiva, no qual verificou-se que, em suas percepções a metodologia os ajudou a estudar individualmente os conteúdos e a praticar habilidades como trabalho em equipe e argumentação.

Teixeira, Botelho e Roncari (2020) corroboram e afirmam que a ABE foi avaliada positivamente por estudantes de medicina e foi eficaz em promover a melhoria do desempenho acadêmico em tópicos de neurofisiologia. Esses resultados sugerem, portanto, que $\mathrm{ABE}$ é uma metodologia de ensino eficiente para auxiliar os estudantes a construir um conhecimento básico sólido.

No entanto, alguns autores encontram resultados discordantes em que o aprendizado em grupo mostrou-se em desfavor do método ABE em relação a outros métodos e pode ter várias justificativas (Masocatto, 2019). Reimschisel et al. (2017) apontam que muitas dimensões da ABE ainda não foram bem estudadas, como variedade dos fatores processuais, participação definida do facilitador e padrão de engajamento dos diversos grupos. Para Bollela et al. (2014) a maioria dos problemas relatados com grupos de aprendizagem (participantes disfuncionais, conflitos entre membros, etc.) é resultado direto de um desenvolvimento inapropriado da própria equipe. Essa etapa, no entanto, é essencial para estimular a discussão em grupo e o ensino-aprendizagem entre os integrantes enquanto esses estabelecem um consenso de resposta e favorece, principalmente, aqueles que estavam de início mais despreparados no começo das atividades.

Um ponto que merece destaque foi a ênfase dada ao feedback frequente, imediato, oportuno e específico durante todo o processo das oficinas. O fornecimento de feedback é fundamental na ABE por seu reconhecido impacto positivo na aprendizagem, na retenção do conhecimento e no desenvolvimento do trabalho em equipe. Ele proporciona a reflexão sobre o que está bom, o que pode ser melhorado e como esta melhora pode ser alcançada (Michaelsen, 2002).

Destaca-se ainda que um mesmo condutor/professor foi suficiente para realizar a atividade com mais de 32 alunos distribuídos em pequenos grupos e foi observado que os participantes assumiram a responsabilidade pelo seu aprendizado e puderam pôr em prática suas habilidades para o trabalho em equipe e, também, de arguição e tomada de decisões. Achados que concordam com o trabalho de Ono et al. (2017) realizado no Japão para verificar os efeitos do ABE na aprendizagem.

Ressalta-se ainda que 96,9\% dos participantes referiram compreender os benefícios da ABE após as oficinas realizadas e, destes, 53,12\% não compreendiam anteriormente. Ganho que foi estatisticamente significante com p valor $<0,05$ e que permite afirmar que as oficinas além de qualificarem os docentes, também foram capazes de sensibilizá-los quanto aos benefícios no processo de ensino-aprendizagem.

Esses dados concordam com estudos prévios em cursos de Farmácia nos Estados Unidos cujos resultados demonstraram que apesar das dificuldades, os docentes percebem que a $\mathrm{ABE}$ incrementa o engajamento e o aprendizado dos alunos (Allen et al., 2013).

Percebe-se que o modelo proposto em tela mostrou-se uma ferramenta com bons resultados no tocante ao aumento de conhecimentos e sensibilização à sua importância, e ainda com grande potencial de desenvolvimento e aperfeiçoamento para que melhor se adeque as realidades locais sem que haja prejuízo ao processo de ensino-aprendizagem.

\section{Conclusão}

Conclui-se, a partir dos resultados, que as oficinas de qualificação foram eficientes para incrementar e qualificar os docentes acerca da metodologia da $\mathrm{ABE}$, apontando para o sucesso das oficinas, o que vem a permitir aos participantes a aplicação dessa estratégia na qualificação do ensino e maior segurança e em suas práticas pedagógicas diárias. 
Ademais, comprovou-se que a tecnologia educacional desenvolvida é uma ferramenta válida para implementação no desenvolvimento docente de outras instituições que desejem implementá-la em suas matrizes curriculares.

Espera-se que esse trabalho venha contribuir para a aprendizagem sobre essa estratégia educacional e que possa tornar acessível a sua aplicação, a fim de estimular docentes e instituições não familiarizados com a ABE a introduzirem esta valiosa ferramenta em seus locais de trabalho. Como sugestão para pesquisas futuras, seria de grande relevância avaliar o aprendizado a longo prazo dos docentes participantes das oficinas de $\mathrm{ABE}$, comparando-se com outros tipos de metodologias.

\section{Referências}

Albuquerque, M. R. T. C. et al. (2021). Team-Based Learning (TBL) como estratégia para o Estudo do Pacto pela Saúde: Relato de experiência na Graduação em Medicina. Brazilian Journal of Health Review, 4(3), 10136-10142.

Albuquerque, M.R.T.C., Martins, A.C.G.S., Caldato, M.C.F, \& Botelho, N.M (2021). Conhecimento de professores sobre aprendizagem em equipe: dificultadores para implantação na graduação em medicina. Pesquisa, Sociedade e Desenvolvimento, 10 (4), e30410414164. https://doi.org/10.33448/rsdv10i4.14164

Allen, R. E. et al. (2013). Aprendizagem em equipe em faculdades e escolas de farmácia dos EUA. Revista Americana de Educação Farmacêutica, 77 (6).

Altintas, L., Altintas, O. \& Caglar, Y. (2014). Uso modificado do aprendizado baseado em equipe em um curso de oftalmologia para estudantes de medicina do quinto ano. Avanços na educação em fisiologia, 38 (1), 46-48.

Balwan, S. et al. (2015). Use of team-based learning pedagogy for internal medicine ambulatory resident teaching. Journal of graduate medical education, 7 (4), 643 .

Bandeira, D. M. A., Silva, M. A. \& Vilela, R. Q. (2017). Aprendizagem Baseada em Equipe. Revista Portal: Saúde e Sociedade, 2 (1), $371-379$.

Barbiero, A. J. C. et al. (2017). Proposta de novas metodologias para o ensino da disciplina de farmacologia nos cursos de medicina. Revista Científica FAGOC-Saúde, 2 (1), 63-69.

Bleske, B. E. et al. (2014). Aprendizagem baseada em equipe para melhorar os resultados de aprendizagem em uma sequência de curso de terapêutica. Revista Americana de Educação Farmacêutica, 78 (1).

Bleske, B. E. et al. (2016). Uma comparação cruzada aleatória de aprendizagem baseada em equipe e formato de palestra sobre resultados de aprendizagem. American Journal of Pharmaceutical Education, 80 (7).

Bollela, V. R. et al. (2014). Aprendizagem baseada em equipes: da teoria à prática. Medicina (Ribeirão Preto), 47 (3), $293-300$.

Boysen-Osborn, M. et al. (2016). Invertendo a sala de aula de suporte cardíaco avançado com aprendizagem baseada em equipe: comparação do desempenho em testes cognitivos para estudantes de medicina na Universidade da Califórnia, Irvine, Estados Unidos. Jornal de avaliação educacional para profissionais de saúde, 13 .

Brasil. (2014). Ministério da Educação. Conselho Nacional de Educação. Câmara de Educação Superior. Resolução No. 3 de 20 de junho de 2014. Institui diretrizes curriculares nacionais do curso de graduação em Medicina e dá outras providências. Diário Oficial da União, Brasília, 23 jun. Seção 1, p. 8-11.

Cunha, C. R. O. B. J., Ramsdorf, F. B. M. \& Bragato, S. G. R. (2019). Utilização da aprendizagem baseada em equipes como método de avaliação no curso de medicina. Revista Brasileira de Educação Médica, 43 (2), 208-215.

Echeto, L. F. et al. (2015). Avaliação da aprendizagem baseada em equipe e instrução tradicional no ensino de conceitos de prótese parcial removível. Journal of Dental Education, 79 (9), 1040-1048.

Huang, Z. et al. (2016). Aprendizagem baseada em equipe modificada em um estagiário de oftalmologia na China. PLoS One , 11 (4), e0154250.

Harakuni, S. et al (2015). Eficácia da aprendizagem baseada em equipe em microbiologia: um estudo de controle não randomizado. Educação para a saúde, $28(1), 41$.

Ibrahim, M. E. (2020). Instrumento de avaliação do aluno de aprendizagem baseado em equipe (TBL-SAI) para avaliar a aceitação dos alunos do TBL em um saudita. Saudi Med J, 41 (5), 542-547.

Ismail, N. A. S. (2016). Eficácia da aprendizagem baseada em equipe no ensino de genética médica para graduandos de medicina. O jornal da Malásia de ciências médicas: $M J M S, 23$ (2), 73.

Jost, M. et al. (2017). Efeitos da aprendizagem adicional baseada em equipe nas habilidades de raciocínio clínico dos alunos: um estudo piloto. Notas de pesquisa BMC, $10(1), 1-7$.

Keshmiri, F. et al. (2016). Validating and assessing the reaction of medical students toward team-based learning. Acta Medica Iranica, 806-811.

Koh, Y. Y. J. et al. (2020). Análise de aprendizagem baseada em equipe: um estudo de caso empírico. Medicina Acadêmica, 95 (6), 872.

Krug, R. R. et al. (2016). O “bê-á-bá” da aprendizagem baseada em equipe. Revista Brasileira de Educação Médica, 40 (4), $602-610$. 
Research, Society and Development, v. 10, n. 10, e23101018381, 2021

(CC BY 4.0) | ISSN 2525-3409 | DOI: http://dx.doi.org/10.33448/rsd-v10i10.18381

Masocatto, N. O. et al. (2019). Percepção de Alunos de Curso de Graduação em Medicina sobre o Team-Based Learning (TBL). Revista Brasileira de Educação Médica, 43 (3), 111-114.

Michaelsen, L. K. (2002). Introdução ao aprendizado baseado em equipe. Aprendizagem baseada em equipe: um uso transformador de pequenos grupos, 27 51 .

Oliveira, B. L. C. A. et al. (2018). Team-based learning como forma de aprendizagem colaborativa e sala de aula invertida com centralidade nos estudantes no processo ensino-aprendizagem. Revista brasileira de educação médica, 42 (4), 86-95.

Ono, S. et al. (2017). Verificação de efeitos de aprendizagem por aprendizagem baseada em equipe. Yakugaku zasshi: Journal of the Pharmaceutical Society of Japan, 137 (11), 1419-1423.

Pereira A. S. et al. (2018). Metodologia da pesquisa científica. UFSM.

Pereira, C. C. S. B. \& Afonso, R. T. L. (2020). Percepção discente sobre aprendizagem baseada em equipes (TBL) e instrução em pares (PI). Brazilian Journal of Development, 6 (1), 4057-4065.

Reimschisel, T. et al. (2017). A systematic review of the published literature on team-based learning in health professions education. Medical Teacher. $10.1080 / 0142159 X .2017 .1340636$

Sakamoto, S. R. et al. (2020). Aprendizagem baseada em equipes: um ensaio clínico randomizado na graduação em enfermagem. Revista Brasileira de Enfermagem, 73 (2).

Silva Junior, G. B. et al. (2017). Team-Based Learning: Successful Experience in a Public Health Graduate Program. Revista Brasileira de Educação Médica, $41(3), 397-401$.

TEAM-BASED LEARNING COLLABORATIVE. Website. http://www.teambasedlearning.org.

Teixeira, A. A. R., Botelho, C. V. \& Roncari, C. F. (2020). Aprendizagem baseada em equipe em neurofisiologia: desempenho e percepção de estudantes de medicina. Rev Med (São Paulo), 99 (3), 236-41.

Thiollent, Michel. (2013). Metodologia da pesquisa-ação. In: Metodologia da pesquisa-ação. 108-108. 